\title{
Diseño e Implementación de una Plataforma Informática CLOUD/GIS de Gestión, Operación y Control de Sistemas Fotovoltaicos de Iluminación
}

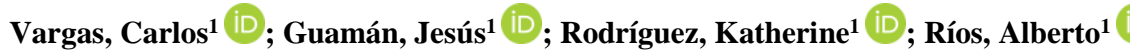 \\ ${ }^{1}$ Universidad Técnica de Ambato, Facultad de Ingeniería en Sistemas, Electrónica e Industrial, Ambato, Ecuador
}

\begin{abstract}
Resumen: En el presente artículo se describe el diseño, simulación e implementación de un prototipo de gestión, operación y control de un sistema de iluminación fotovoltaico desde una plataforma Cloud/GIS. La plataforma Cloud/GIS regula la intensidad lumínica de diferentes luminarias LED interconectadas y visualizadas en un Sistema de Información Geográfico, GIS. El prototipo electrónico de iluminación LED se instaló en la Universidad Técnica de Ambato. El prototipo de control automático de iluminación se programa para dos formas de funcionamiento: los días de lunes a viernes, y los sábados y domingos. Asimismo, se propone una metodología de análisis del impacto económico y medioambiental, asociado a la implementación de una plataforma de gestión, operación y control de sistemas fotovoltaicos de iluminación. Finalmente, se realizó un análisis comparativo del impacto económico y medioambiental de sistemas convencionales de alumbrado público, sin ningún tipo de control de luminosidad, frente a un sistema de alumbrado público integrado a una plataforma Cloud/GIS de gestión, operación y control de luminarias LEDs.
\end{abstract}

Palabras clave: Alumbrado público, Cloud Computing, control, GIS, iluminación fotovoltaica, LEDs.

\section{Design and Implementation of CLOUD/GIS Platform for Management, Operation and Control of Photovoltaic Lighting Systems}

\begin{abstract}
This article describes the design, simulation and implementation of a prototype for the management, operation and control of a photovoltaic lighting system from a Cloud / GIS platform. The Cloud / GIS platform controls the luminosity level of LED luminaires and integrates a Geographic Information System, GIS. The electronic prototype of LED lighting was installed at the Technical University of Ambato. The automatic lighting control prototype is programmed for two forms of operation: the days from Monday to Friday, and Saturdays and Sundays. Likewise, a methodology for analyzing the economic and environmental impact associated with the implementation of a platform for the management, operation and control of photovoltaic lighting systems is proposed. Finally, a comparative analysis of the economic and environmental impact of conventional public lighting systems was carried out, without any control of luminosity, in front of a public lighting system integrated to a Cloud / GIS platform for the management, operation and control of LED luminaires.
\end{abstract}

Keywords: Public lighting, Cloud Computing, Control, Photovoltaic Lighting, LEDs.

\section{INTRODUCCIÓN}

El servicio de Cloud Computing como plataforma informática es una tecnología que permite al usuario disponer de aplicaciones informáticas propias y personalizadas en la infraestructura de la nube, sin necesidad de grandes y costosas instalaciones, y un acceso seguro a la información

*a.rios@uta.edu.ec

Recibido: 25/09/2018

Aceptado: 04/05/2020

Publicado: $31 / 05 / 2020$

10.33333/rp.vol45n2.02

CC BY 4.0 desde cualquier parte del mundo, únicamente, con una conexión a internet (Morales, M. F., 2012).

En el presente artículo se propone integrar los servicios Cloud Computing con aplicaciones GIS en una arquitectura multitareas y multipropósitos denominada Cloud/GIS. En este sentido, se pueden crear aplicaciones web para el manejo de información de manera geográfica, desde la nube. Asimismo, facilita la localización de diferentes flujos de trabajo en una amplia gama de procesos e industrias. Entre las diferentes aplicaciones desplegadas por los sistemas GIS interconectados a internet destacan: inventario de árboles e 
inspección de trabajos forestales, gestión de recursos hídricos y cuencas hidrográficas, seguridad pública y respuesta de emergencias, obras viales, ciudades inteligentes, mapas públicos de la ciudad, etc. (Manzano, S., Peña-Ortiz, R., Guevara, D., \& Ríos, A. 2014; Rodríguez, N. R., Murazzo, M. A., Chávez, S. B., \& Guevara, M. J, 2014; Segura, A. A. 2016; Gómez Delgado, M., 2016).

Por otra parte, existen trabajos asociados a innovadoras plataformas de comunicaciones y tecnologías de la información que incluyen nuevos elementos en su estructura (Guamán, J. I., Vargas, C. L., García, M. G., \& Ríos, A. 2017; Vargas, C., Guamán, J., Nogales, R., \& Ríos, A. 2017).

Las plataformas Cloud permiten consultar datos y visualizar información de manera remota, una de las funcionalidades de las plataformas Cloud es su fácil y amplia capacidad de expansión e integración de información por ejemplo sistemas fotovoltaicos de generación eléctrica (Guamán, J., Guevara, D., Vargas, C., Ríos, A., \& Nogales, R. 2017; Manzano, S., Peña, R., Guevara, D., \& Ríos, A. 2014).

Además, las plataformas Cloud/GIS son herramientas óptimas para la gestión, operación y control de los sistemas de iluminación públicos. El objetivo de implementar plataformas Cloud/GIS en los sistemas de iluminación públicos se aplica la optimización de la gestión del alumbrado, así como la reducción del consumo de energía y las emisiones contaminantes. Las plataformas Cloud/GIS han incursionado en el mundo de los negocios definiéndose como un modelo de prestación de servicios, permitiendo a los usuarios acceder a los diferentes servicios y necesidades de una forma sencilla y flexible. Cloud/GIS es una herramienta informática gestionada desde la web, con un coste económico de operación mínimo. Además, el acceso al control y manejo de la información es bastante sencilla (Pisu, C., \& Casu, P., 2013). La aplicación de las herramientas Cloud/GIS en la gestión, operación y control de los sistemas de iluminación pública es un tema que despierta un gran interés tanto para las empresas desarrolladoras de software como para las compañías eléctricas y los organismos públicos (Dangermond, J., 2012; Mahmoud, E., Hegazy, O., \& ElDien, M. N., 2013; Ríos, A., Vargas, C., Guamán, J., \& Rodríguez, K. 2017).

Actualmente, las investigaciones asociadas a las plataformas de control de sistemas de iluminación se centran en la implementación de técnicas de control del flujo luminoso de las luminarias, así como la aplicación de innovadoras tecnologías de adquisición y transmisión de la información. En los siguientes párrafos se describen algunos de los más innovadores sistemas de gestión, operación y control.

- En el año 2016, en Chicago, Estados Unidos, el Departamento de Ciencias de la Computación del Instituto de Tecnología implementó un sistema de iluminación público basado en una red inalámbrica y tecnología de control centralizado, como una aplicación para ciudades inteligentes, con el objetivo de reducir costos de energía y mejorar la seguridad pública. El sistema piloto de control de iluminación integra 18 luminarias inteligentes en una red tipo malla. Las luminarias tienen la capacidad de enviar información acerca de su consumo actual, luminosidad y potencia. Asimismo, se puede controlar el porcentaje de atenuación de las luminarias en incrementos de $10 \%$ a través de una API, Application Programming Interface (Jin, D., Hannon, C., Li, Z., Cortes, P., Ramaraju, S., Burgess, P. \& Shahidehpour, M. 2016). La Figura 1, muestra el esquema del sistema piloto de iluminación propuesto.

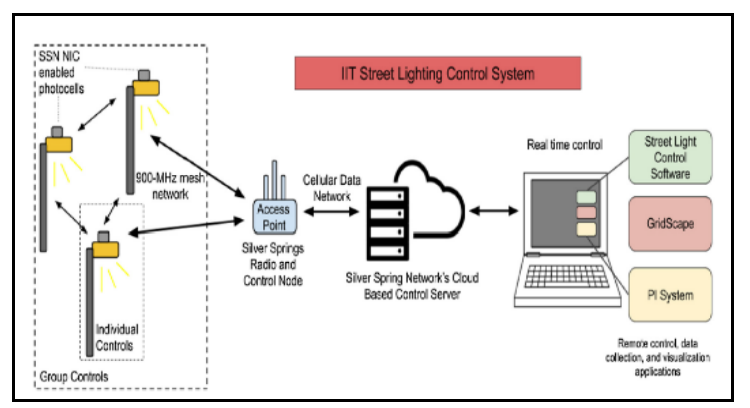

Figura 1: Sistema piloto centralizados de iluminación público (Jin, D., Hannon, C., Li, Z., Cortes, P., Ramaraju, S., Burgess, P. \& Shahidehpour, M. 2016)

- En el año 2016, en Calicut, India, el Departamento de Diseño de Sistemas Embebidos, NIELIT, presentó el diseño e implementación de un sistema integrado de control de iluminación para incrementar el ahorro energético en el alumbrado público. El sistema propuesto utiliza sensores infrarrojos para encender las luminarias al momento de detectar la aproximación de vehículos. La propuesta se implementó en un sistema integrado de control de iluminación público y se basa en la detección de vehículos y/o cualquier otro obstáculo. La información en tiempo real del estado de las luminarias se encuentra desarrollada en una Plataforma de Conectividad Inteligente, PCI, y puede ser visualizada en cualquier momento (Parkash, Prabu V, Dandu Rajendra, 2016). La Figura 2, muestra el diagrama de bloques empleado para el desarrollo del sistema de inteligente de iluminación público basado en una Plataforma de Conectividad Inteligente. 


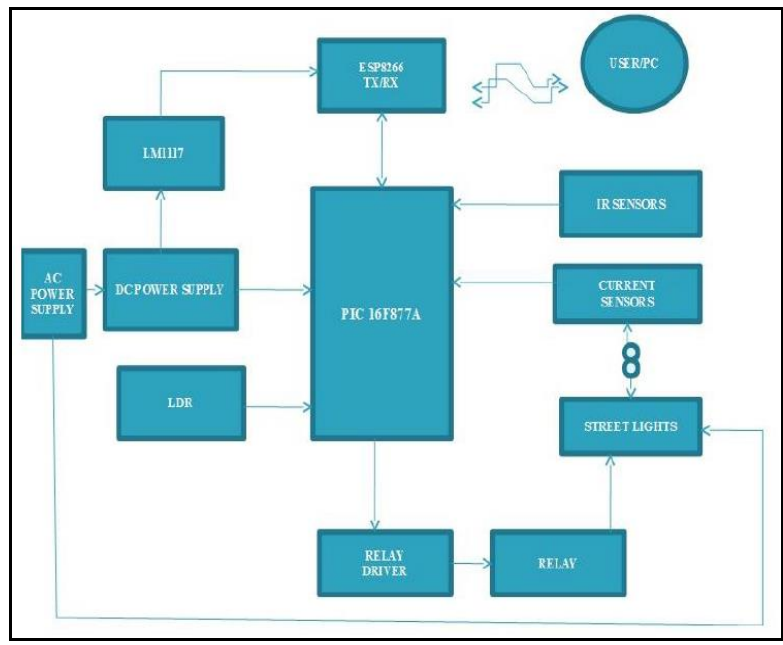

Figura 2: Diagrama de bloques del sistema inteligente de iluminación público basado en una Plataforma de Conectividad Inteligente (Parkash, Prabu V, Dandu Rajendra, 2016).

- En el año 2016, en Bangalore, Karnataka India, se desarrolló un prototipo automatizado para el control de un sistema de iluminación pública de luminarias LED. El prototipo desarrollado se centra en la integración de la tecnología denominada Internet de las Cosas, IoT, que controla la intensidad luminosa de las luminarias, con ayuda de un sistema electrónico de conmutación, en función de la cantidad de luz natural y de una programación temporizada de actividades. Los sensores empleados se conectan de forma remota a internet y los datos registrados se almacenan para su análisis y visualización (Soni, Soni, \& Annapurna, 2016).

- Finalmente, se presenta un análisis comparativo de un sistema de iluminación convencional frente al nuevo prototipo de iluminación propuesto, que integra un sistema de control de la intensidad luminosa de las luminarias LED (Jin, D., Hannon, C., Li, Z., Cortes, P., Ramaraju, S., Burgess, P., \& Shahidehpour, M., 2016). La figura 3, muestra el diagrama de bloques del sistema propuesto.

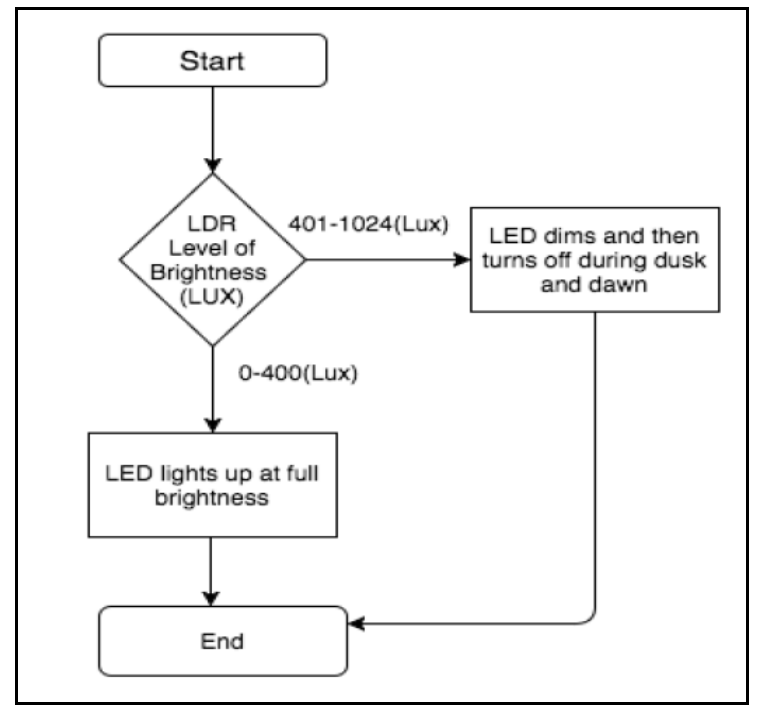

Figura 3: Diagrama de bloques del sistema de control remoto automatizado de luminarias LED (Jin, D., Hannon, C., Li, Z., Cortes, P., Ramaraju, S., Burgess, P., \& Shahidehpour, M., 2016).

\section{PROTOTIPO DE CONTROL DE ILUMINACIÓN DESDE UNA PLATAFORMA CLOUD/GIS.}

El desarrollo del prototipo propuesto se realizó en función de las siguientes etapas:

- Descripción general del funcionamiento del prototipo.

- Diseño del circuito de control de intensidad.

- Programación del microcontrolador.

- Implementación y validación del circuito regulador.

- Sistema de comunicación inalámbrica.

- Diseño y programación de la interfaz web.

- Integración del sistema de información geográfica, GIS.

\subsection{Descripción General del Prototipo.}

Las plataformas Cloud permiten la integración de diferentes tecnologías de comunicación. Una de las tecnologías más robustas y altas prestaciones es la comunicación inalámbrica (Manzano, S., Peña, R., Guevara, D., \& Ríos, A. 2014; Manzano, S., Guevara, D., \& Ríos, A. 2015)

En la Universidad Técnica de Ambato se ha implementado una plataforma Cloud/GIS de control de sistemas renovables de iluminación, basados en tecnología LED, con control inteligente del flujo luminoso. El prototipo controla una luminaria LED de $24 \mathrm{~W}$ de potencia nominal.

El control de iluminación se puede realizar de forma manual o de forma automática, con ayuda de un sensor detector de luz natural que determina el inicio del funcionamiento del sistema de iluminación. El funcionamiento de la plataforma Cloud/GIS se basa en el control del porcentaje de iluminación a diferentes horas por medio de un control por modulación de ancho de pulso, en inglés PWM (Pulse Width 
Modulation). La modulación por ancho de pulso controla la corriente del LED. Además, se realiza la adquisición y tratamiento de la información de la operación del sistema de iluminación para su posterior visualización y análisis. Asimismo, se incluye un sensor de presencia que maximiza la intensidad luminosa al detectar la presencia de personas.

La propuesta tiene como aspecto innovador la integración de un Sistema de Información Geográfica, GIS, de la ubicación de luminarias LED en una plataforma Cloud, que permite la gestión, operación y control en diferentes zonas del sistema de alumbrado público. La programación de la plataforma Cloud se realiza con ayuda de hardware y software libre, mientras que la programación de la interfaz web se basa en lenguaje PHP. El control del nivel de iluminación se realiza con ayuda de un microcontrolador, que trabaja en función de la información de presencia y nivel de iluminación natural. En la Figura 4, se presenta el esquema general del funcionamiento de la plataforma Cloud/GIS de gestión, operación y control de sistemas de iluminación fotovoltaicos.

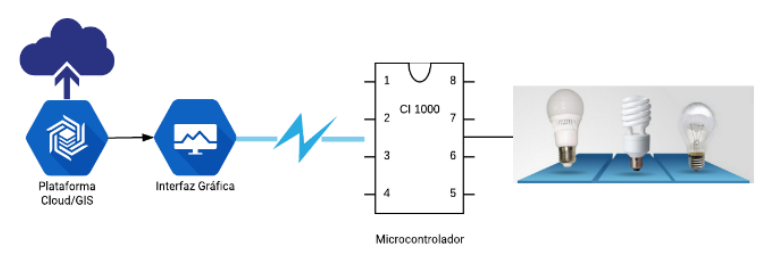

Figura 4: Esquema general del funcionamiento de la plataforma Cloud/GIS de gestión, operación y control de sistemas de iluminación fotovoltaicos.

En la Figura 5, se presenta el control de encendido y apagado de las luminarias a través de un control automático programado en una interfaz web. De esta manera se despliegan gráficas del funcionamiento del sistema por un día específico, permitiendo al usuario elegir un historial del comportamiento del sistema de iluminación. Por otra parte, también es factible desplegar información en tiempo real de los valores de porcentaje actual en la luminaria y ahorro energético.

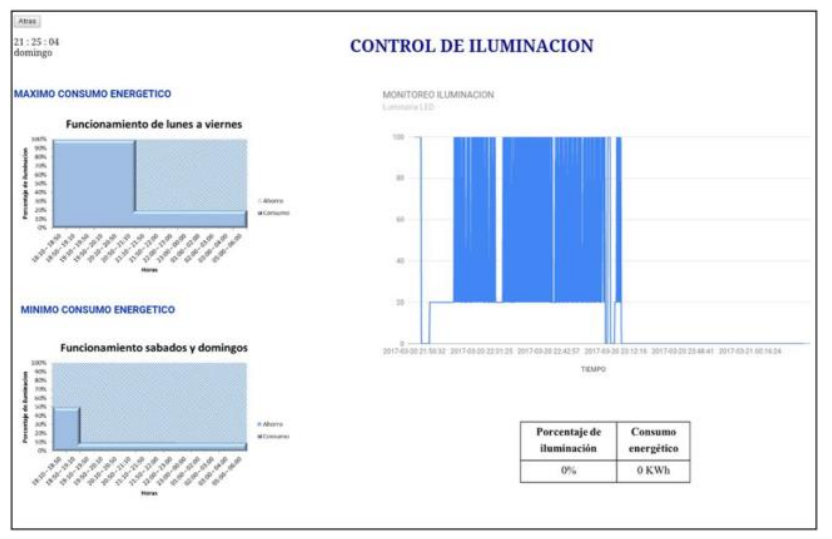

Figura 5: Interfaz web del control automático
Asimismo, el control de iluminación integra un sistema de georreferenciación de las luminarias empleando librerías de OpenLayers, que se complementa con las bibliotecas de JavaScript y PHP para interactuar, acceder y visualizar mapas en diferentes navegadores web.

\subsection{Diseño del Circuito de Control de Intensidad.}

El prototipo de control de las luminarias LEDs funciona en corriente continua. El sistema de control requiere modular el valor de la corriente continua de entrada, con ayuda de un regulador lineal de corriente o un convertidor DC-DC. En la Figura 6, se visualiza la simulación del regulador lineal de corriente, que se basa en la comparación del nivel de voltaje aplicado a la entrada del circuito para regular la tensión en los terminales que conectan con la luminaria.

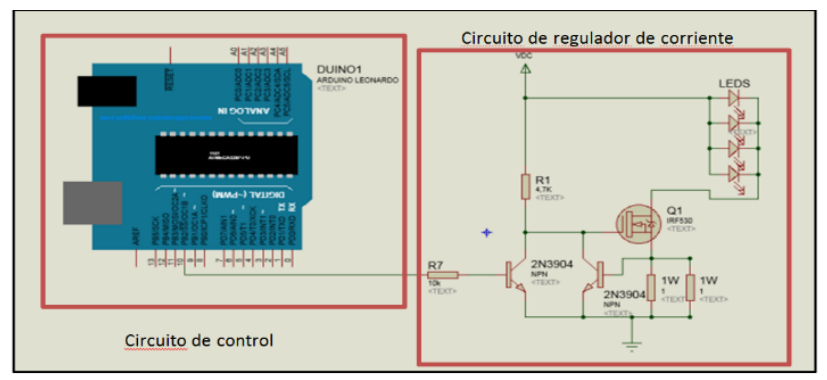

Figura 6: Circuito de control y circuito regulador de corriente DC

El regulador lineal de corriente continua está compuesto por un transistor del tipo MOSFET de potencia de canal $\mathrm{N}$, el MOSFET IRF530 y un par de transistores 2N3904, conectados a la compuerta del MOSFET. Uno de los transistores 2N3904, se conecta a la salida de control; es decir, al microcontrolador. De igual manera, se requiere de un conjunto de resistencias conectadas en paralelo. El conjunto de resistencias se conecta en serie con el LED, regulando la caída de tensión al encender el LED.

En la Figura 5, se observa el circuito controlador de tipo serial, que regula la intensidad a través del MOSFET, del transistor y del conjunto de resistencias conectadas en paralelo. Al encender el circuito, el MOSFET se energiza al detectar una caída de tensión positiva en la compuerta conectada a la resistencia de 4,7 $\mathrm{K} \Omega$. La conducción del MOSFET enciende los LEDs y produce una caída de tensión en las resistencias conectadas en paralelo. El aumento de la intensidad genera un aumento de tensión aplicada al conjunto de resistencias. Si se supera la tensión umbral del transistor, $0.6 \mathrm{~V}$, el transistor conduce y disminuye la tensión aplicada en el MOSFET, inyectando así en los LEDs una intensidad proporcional al valor de las resistencias.

La luminaria LED se compone de una matriz de 8 LEDs de 3 $\mathrm{W}$ cada uno a una tensión de 3,7 V. La intensidad que circula por el LED a potencia nominal es igual a 0,81A. La intensidad nominal define el valor de la resistencia que 
permite regular el nivel de iluminación. El valor de resistencia requerido es $0,7 \Omega$, pero no existen esas resistencias, por tanto, se optó por utilizar dos resistencias en paralelo de $1 \Omega$ que equivalen a $0,5 \Omega$.

\subsection{Programación del Microcontrolador}

A la entrada del transistor 2N3904 se conecta un microcontrolador de la familia Arduino, específicamente un Arduino Leonardo. La programación del microcontrolador se encarga de regular el flujo luminoso de las lámparas, a través de un control PWM. En corriente continua, la señal de tensión no varía con el tiempo. El control PWM divide la señal de tensión en ciclos de trabajo y modula el ancho del pulso de la señal. La duración del periodo de tiempo es inversa a la frecuencia PWM, que para el microcontrolador empleado es igual a $500 \mathrm{~Hz}$; es decir, 2 ms (Dangermond, J., 2012).

En la programación del microcontrolador, los niveles de iluminación se deben ajustar a un rango de valores que oscila entre 0 a 255 . Un cero corresponde a un nivel de iluminación del $100 \%$ mientras que el valor de 255 corresponde al $0 \%$.

En la programación del microcontrolador se establecieron diez valores, entre 0 a 255, que permiten obtener escalones de control de $10 \%$, entre el $10 \%$ al $100 \%$ del total de la potencia entregada por el LED.

\subsection{Implementación y Validación del Circuito Regulador}

El LED utilizado es un LED FLOOD LIGHT OUTDOOR de $24 \mathrm{~W}$. En su interior tiene un LED DRIVER, que convierte la corriente alterna en corriente continua. El controlador del LED se conecta a la placa del circuito de control de intensidad luminosa del LED. En la Figura 7, se muestra la implementación del circuito de control de la luminaria LED.

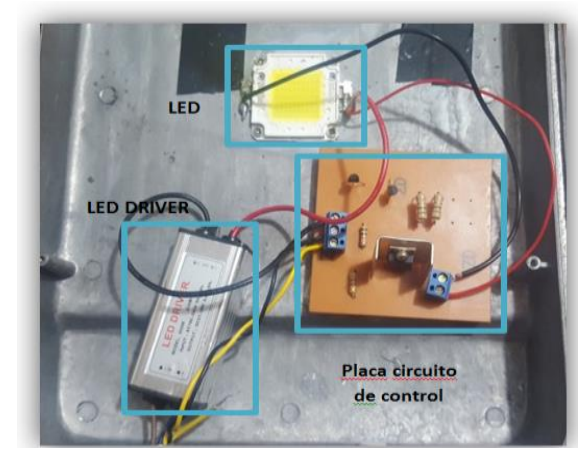

Figura 7: Circuito de control de la intensidad luminosa, implementado en el interior de la luminaria LED

Según datos del fabricante, la luminaria LED se alimenta a una tensión nominal de 120 Vac y consume 0.2 A, equivalente a un consumo de potencia igual a $24 \mathrm{~W}$. En el lado de corriente continua, la tensión de salida del controlador interior del LED oscila entre $21 \mathrm{Vdc}$ y $36 \mathrm{Vdc}$, consumiendo una intensidad de 0,6 A. Para validar el comportamiento del circuito de control se realizaron las correspondientes pruebas de funcionamiento. Las pruebas consistieron en encender la luminaria a diferentes porcentajes de iluminación. Asimismo, se midieron los valores de tensión y corriente consumidos por la luminaria para el cálculo de valor de potencia eléctrica.

En la Tabla 1, se muestran los valores de tensión, intensidad y potencia medidos en el proceso de validación del circuito de control de luminosidad del LED.

Tabla 1: Valores de potencia a diferentes porcentajes de nivel de luminosidad.

\begin{tabular}{cccc}
\hline \hline Porcentaje $(\%)$ & Voltaje $(\mathbf{V})$ & Intensidad $(\mathbf{A})$ & Potencia $(\mathbf{W})$ \\
\hline 0 & 0 & 0 & 0 \\
10 & 5,5 & 0,397 & 2,18 \\
20 & 8,3 & 0,497 & 4,13 \\
30 & 11,4 & 0,567 & 6,46 \\
40 & 13,8 & 0,596 & 8,22 \\
50 & 16,8 & 0,625 & 10,50 \\
60 & 18,9 & 0,643 & 12,15 \\
70 & 26,1 & 0,601 & 15,69 \\
80 & 27,7 & 0,603 & 16,70 \\
90 & 30,4 & 0,606 & 18,42 \\
100 & 33,6 & 0,609 & 20,46 \\
\hline \hline
\end{tabular}

\subsection{Modalidad de la Comunicación.}

El circuito de control es implementado en la parte interior de la luminaria cerca del LED DRIVER. En este sentido, se empleó un sistema de comunicación inalámbrica basado en el estándar IEEE 802.11 que especifica las normas de funcionamiento de una red de área local inalámbrica (WLAN) (Parkash, Prabu V, Dandu Rajendra, 2016).

El sistema de comunicación inalámbrica permite comunicar el control de iluminación con la plataforma Cloud/GIS. El núcleo del sistema de comunicación está conformado por una tarjeta Shield Arduino YUN, que es compatible con Arduino Leonardo conformando así un conjunto de dispositivos electrónicos que controlan, reciben y envían datos desde y hacia la plataforma Cloud/GIS. La tarjeta Shield Arduino YUN, procesa la información en tiempo real y la almacena en una base de datos previamente instalada. Además, una de las principales funcionalidades de esta tarjea es su compatibilidad con diferentes sensores, asimismo, integra un socket que permite colocar una tarjeta microSD que serviría como respaldo de la información que se procesa.

\subsection{Diseño y Programación de la Interfaz Web}

El diseño y programación de la interfaz web se realizó sobre un servidor apache, instalado como una instancia en la Plataforma OpenStack de la Facultad de Ingeniería en Sistemas, Electrónica e Industrial, FISEI. PHP es un lenguaje 
de programación de código abierto muy popular y que permite incluir contenido HTML, ideal para diseñar interfaces web. Una de las ventajas de utilizar PHP es el manejo de peticiones web que le permitirá al usuario registrar información en la base de datos de forma manual. De igual forma, mediante extensiones es posible la creación de archivos de diferentes formatos para generar reportes. El diseño de la interfaz permite el control de la iluminación de forma manual o automática. En el primer caso, en forma manual se ha diseñado un panel de control que permite enviar diferentes valores correspondientes al nivel del flujo luminoso de las luminarias. Por otra parte, el funcionamiento de forma automática se realiza a través de un reloj implementado en el servidor, el reloj marcará el tiempo de inicio y final del sistema de iluminación. La Figura 8, muestra la apariencia visual del diseño de la interfaz web implementada.

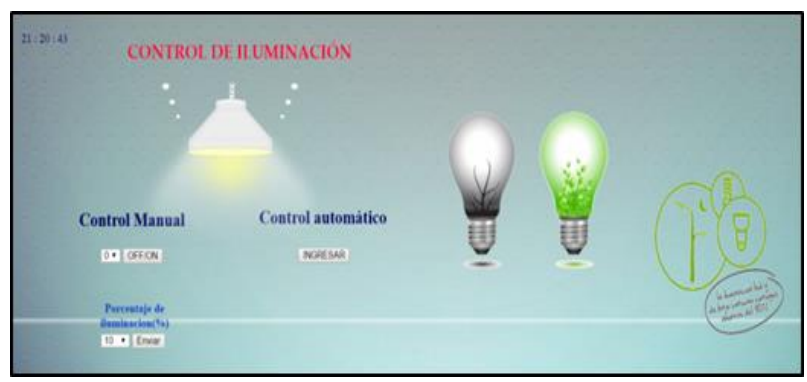

Figura 8: Diseño de la interfaz web para el control de la iluminación.

El control manual permite elegir el porcentaje de iluminación deseado para la luminaria, igualmente, apagarla y/o encenderla. El dato enviado desde la interfaz web se registra en una base de datos instalada en el servidor y se actualiza cada vez que se envíe un nuevo valor. Este valor es recibido por el microcontrolador, encargado de enviar la orden al circuito de control de intensidad, que enciende la luminaria LED al porcentaje de iluminación deseado. El control automático de iluminación permite que la luminaria LED cambie el nivel de iluminación durante la noche de forma automática, integrando un sensor de luz, que establece el inicio del control de iluminación. El prototipo de control del nivel de iluminación recibe suministro eléctrico desde un sistema solar fotovoltaico, instalado en el tejado de la FISEI en la Universidad Técnica de Ambato.

\subsection{Integración del Sistema de Información Geográfica, GIS.}

El control de iluminación integra un Sistema de Información Geográfica, GIS, que permite la localización geográfica de las luminarias, controladas automáticamente desde la web. Para la integración del GIS se utilizó OpenLayers, una biblioteca de JavaScript de código abierto que garantiza el acceso, gestión y visualización de mapas de manera interactiva, en la mayoría de navegadores web (Jin, D., Hannon, C., Li, Z., Cortes, P., Ramaraju, S., Burgess, P., \& Shahidehpour, M. 2016).
La clase OpenLayers.Map crea el mapa a visualizar en la pantalla. Para cargar el mapa en la zona deseada, en este caso, asociada a la Universidad Técnica de Ambato, UTA, se establecen las coordenadas de la zona indicada. Las coordenadas de la UTA son latitud: -1.2690897 y longitud: 78.6257485. Al mapa añadido se agrega una capa de información mediante la clase OpenLayers.Layer. El empleo del método addLayer agrega la capa de información al mapa (Jin, D., Hannon, C., Li, Z., Cortes, P., Ramaraju, S., Burgess, P., \& Shahidehpour, M. 2016). De esta manera, el usuario visualiza información sobre el sentido de circulación y los nombres de las calles. Se utilizó la clase OpenLayersMap porque presenta información geográfica más detallada del campus universitario. Así, en la interfaz GIS se puede visualizar el nombre de la Facultad y los sitios de parqueadero que son alumbrados por las luminarias.

Asimismo, se colocaron marcadores para visualizar en el mapa la ubicación de la luminaria LED integrada al prototipo de control de iluminación, la misma que se encuentra ubicada en la FISEI, UTA. En este sentido, es posible acceder remotamente a través de la interfaz web y controlar el nivel de iluminación. La Figura 9, muestra la interfaz web de la conexión entre el sistema GIS y el servidor web.

\section{UNIVERSIDAD TECNICA DE AMBATO}

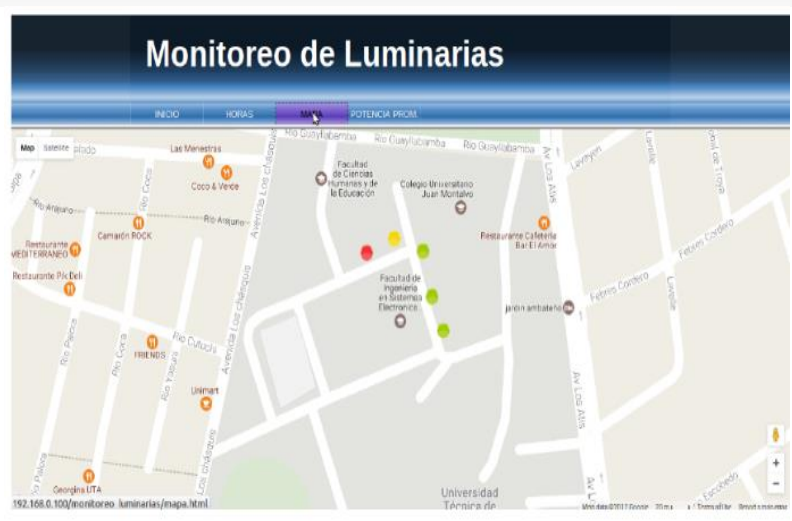

Figura 9: Interfaz web de localización geográfica de la luminaria integrada al control de iluminación

\section{CONDICIONES DE FUNCIONAMIENTO DE LA PLATAFORMA CLOUD/GIS EN LA UTA}

Para la definición de las condiciones de funcionamiento de la plataforma Cloud/GIS se realizó un registro de la afluencia de personas que transitan en horas de la noche, en el exterior de la FISEI. El registro de tránsito de personas se realizó entre las 18:00, hora de inicio de funcionamiento del sistema de iluminación exterior, y las 22:00 de la noche, hora de cierre de la universidad, Tabla 2. 
Tabla 2: Datos del registro de afluencia de personas entre 18:00 y 22:00

\begin{tabular}{cc}
\hline \hline Tiempo & Personas \\
\hline 18:00 - 18:10 & $\sim 98$ \\
18:10-18:50 & $\sim 148$ \\
18:50-19:10 & $\sim 592$ \\
19:10-19:50 & $\sim 148$ \\
19:50-20:10 & $\sim 113$ \\
20:10-20:50 & $\sim 125$ \\
$20: 50-21: 10$ & $\sim 430$ \\
$21: 10-21: 50$ & $\sim 24$ \\
$21: 50-22: 00$ & $\sim 8$ \\
$22: 00-6: 00$ & $\sim 8$ \\
\hline \hline
\end{tabular}

El análisis del registro de afluencia de personas permitió determinar las condiciones de operación, gestión y control de los niveles de iluminación de la plataforma Cloud/GIS, durante la noche. En función de la regulación No. CONELEC 005/2014, que regula los parámetros fotométricos para áreas peatonales y de tráfico de baja velocidad, como sucede en los exteriores de la FISEI, de esta manera se establecieron dos tipos de iluminación para diferentes horarios.

El horario comprendido entre los días lunes a viernes se rige a una clase de iluminación tipo P2, donde el valor promedio de iluminación horizontal es de 10 luxes y un valor mínimo de 2 luxes. Por otra parte, para los días sábados y domingos, se establece una clase de iluminación tipo P3, con un valor de iluminación horizontal promedio de 7.5 luxes y un valor mínimo de 1.5 luxes (Ríos, A., Vargas, C., Guamán, J., \& Rodríguez, K., 2017).

En este sentido, en las Tablas 3 y 4, se muestran los valores de niveles de iluminación óptimos en el transcurso de la noche. El sensor detecta ausencia de luz natural y activa el sistema de iluminación, con un nivel de iluminación del $100 \%$ hasta las 21:10 horas, asociada a la mayor afluencia de personas. A partir de las 21:10 horas, el nivel de iluminación se reduce al 20\% durante el resto de la noche hasta las 6 am, hora en la que se desactiva el sistema de iluminación, como se puede apreciar en la Tabla 3.

Tabla 3: Niveles de iluminación de lunes a viernes.

\begin{tabular}{ccc}
\hline \hline Tiempo & Personas & $\begin{array}{c}\text { Nivel de } \\
\text { Iluminación }\end{array}$ \\
\hline 18:00-21:10 & $\sim 1686$ & $100 \%$ \\
21:10-6:00 & $\sim 8$ & $20 \%$ \\
\hline \hline
\end{tabular}

En la Tabla 4, se visualizan los horarios de operación del sistema de iluminación para los fines de semana. El sistema inicia su funcionamiento con un nivel de iluminación equivalente al $50 \%$ de potencia nominal que permanecerá sin variación hasta las 19:10. A partir de las 19:10, el nivel de iluminación se reduce al $10 \%$ de la potencia nominal de la luminaria LED que permanecerá sin variación hasta el amanecer, horario en el que se registra aproximadamente la circulación de dos personas por hora.

Tabla 4: Niveles de iluminación de sábado a domingo.

\begin{tabular}{ccc}
\hline \hline Tiempo & Personas & Porcentaje \\
\hline 18:10 - 19:10 & $\sim 15$ & $50 \%$ \\
19:10-6:00 & $\sim 23$ & $10 \%$ \\
\hline \hline
\end{tabular}

La definición de las condiciones de funcionamiento permite establecer escenarios máximos y mínimos de consumo energético, en función de los horarios expuestos.

Así, en la Figura 10, se observa el consumo de energía eléctrica en color azul, mientras que, en color blanco-azul, se visualiza el ahorro de energía, que se obtiene al implementar el control del sistema de iluminación supervisado por la plataforma Cloud/GIS. De lunes a viernes, la plataforma Cloud/GIS garantiza un nivel de iluminación máximo, con ayuda de un sensor de luz, y un nivel de iluminación mínimo, con ayuda de un sensor de presencia. Los sábados y domingos, la plataforma Cloud/GIS, actuará en función de los dos sensores el de luz y el de detección de personas.

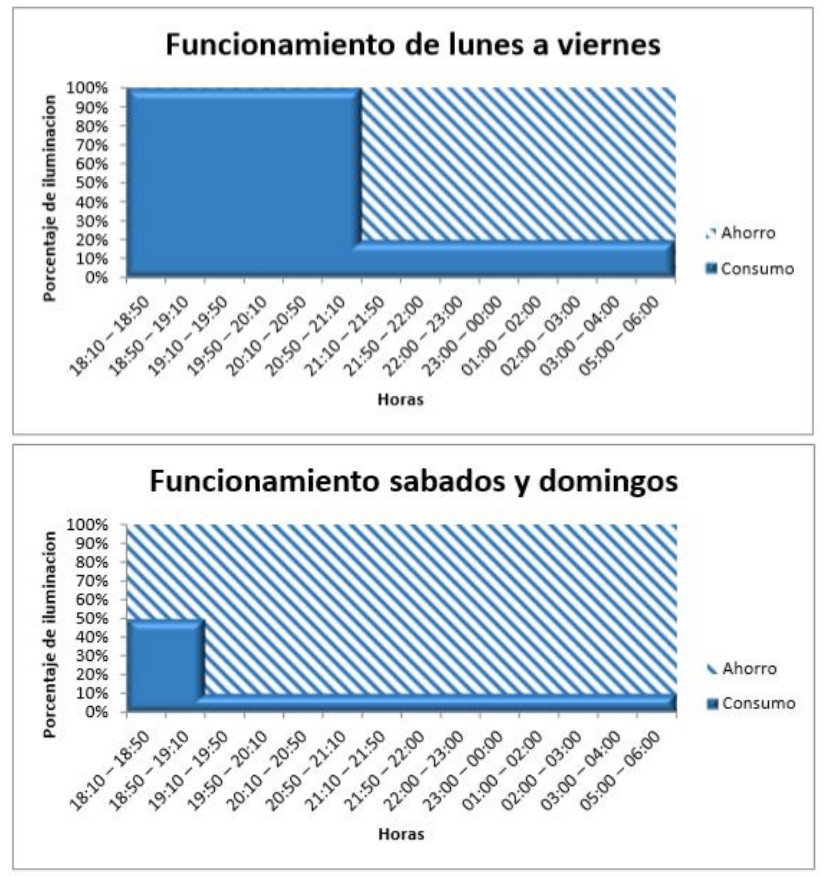

Figura 10: Condiciones de funcionamiento del control de iluminación.

Para la programación de la plataforma Cloud/GIS se determina el tiempo inicial de funcionamiento. El sensor de luz se activa entre las 18:10 y 18:20, en función de la luminosidad del ambiente. Los fines de semana, el nivel de iluminación es del 50\% durante 1 hora y a un 10\% durante el resto de la noche, debido a la poca afluencia de personas en esos días. 
La programación y visualización de un reloj en la página web, con la hora y el día actual del servidor, permite que el valor de porcentaje de iluminación de la luminaria cambie de forma automática, enviando este valor a la base de datos y posteriormente al microcontrolador encargado de dar la orden a la luminaria de cambiar su porcentaje de iluminación. El sensor de presencia permite incrementar el nivel de iluminación al detectar la presencia de algunas personas.

Asimismo, es importante presentar escenarios de operación del sistema de iluminación. Existen diferentes formas de representar escenarios de iluminación como lo que se presenta en el análisis de operación de luminarias en autopistas inteligentes (Vargas, C., García, M., Guevara, D., \& Ríos, A. 2016).

\section{METODOLOGÍA DE EVALUACIÓN DEL IMPACTO ECONÓMICO Y MEDIOAMBIENTAL}

La metodología propuesta de evaluación del impacto económico y medioambiental de la implementación del prototipo propuesto se basa en el trabajo presentado por (Vargas, C., Guevara, D., \& Ríos, A. 2014). En este sentido, inicialmente, se evalúa el sistema de iluminación de vapor de mercurio actualmente existente en comparación con el funcionamiento del sistema de iluminación LED implementado durante un día. Posteriormente, se analiza el impacto de la implementación del sistema de control inteligente en un horizonte de 1 y 10 años, respectivamente.

\subsection{Descripción de la Metodología de Evaluación para Diferentes Escenarios de Iluminación.}

Las luminarias LED presentan un mayor rendimiento en la iluminación pública y mejores características técnicas frente a otras tecnologías de iluminación como lámparas incandescentes, halógenas, fluorescentes compactas y la mayoría de las fluorescentes lineales (Yoomak, Jettanasen, Ngaopitakkul, Bunjongjit, \& Leelajindakrairerk, 2018). En la actualidad, la tecnología LED es considerada como la mejor alternativa para incrementar la eficiencia energética en los sistemas de iluminación.

La metodología propuesta de evaluación del impacto económico y medioambiental de la integración de sistemas de control de iluminación se basa en las siguientes etapas:

- Cálculo del consumo energético diario y anual de luminarias convencionales - vapor de sodio e incandescentes - y luminarias LED.

- Cálculo del coste económico anual de iluminación pública en luminarias convencionales - vapor de sodio e incandescentes - y luminarias LED.
- Cálculo del sistema de iluminación, incluyendo el costo de las luminarias y el costo de los dispositivos de control.

- Cálculo del costo del sistema de iluminación en un horizonte de 10 años.

- Cálculo de la cantidad de emisiones de $\mathrm{CO}_{2}$ por parte de las luminarias evaluadas.

Para la evaluación del impacto económico y medio ambiental, empleando la metodología propuesta, se consideran luminarias de vapor de sodio y luminarias LED. La metodología permite evaluar el ahorro de consumo energético y económico al reemplazar luminarias convencionales por luminarias LED. También, es necesario evaluar el ahorro energético y económico que se obtendría al implementar la plataforma Cloud/GIS. La metodología propuesta en un inicio permite evaluar dos escenarios de iluminación: de mínimo y máximo ahorro energético.

El escenario de mínimo ahorro energético corresponde al funcionamiento de las luminarias - vapor de sodio y LED sin ningún control del nivel de iluminación. El máximo ahorro energético corresponde al funcionamiento de las luminarias LED, basado en el control de iluminación implementado en la plataforma Cloud/GIS. Adicionalmente, se presenta la evaluación del ahorro energético que se podría obtener con la implementación del prototipo de control de iluminación en lámparas de vapor de sodio del Campus Huachi de la UTA y en las luminarias incandescentes de los reflectores de la FISEI, respectivamente.

Por otra parte, en Ecuador los costes de alumbrado público se determinan por tarifa y rango de consumo. En la Universidad Técnica de Ambato, el tipo de servicio de alumbrado público es denominado como beneficio público, que de acuerdo al pliego tarifario vigente se le asigna a escuelas, colegios y universidades públicas, con un rango de consumo eléctrico por hora de orden superior, con una tarifa de 0,063 USD/KWh mensual para la zona de Ambato (Pliego Tarifario 2018).

\subsection{Escenario de Máximo y Mínimo Ahorro Energético}

Para la evaluación del escenario de máximo y mínimo ahorro energético se han considerado luminarias de vapor de sodio de $250 \mathrm{~W}$ y luminarias LED de $24 \mathrm{~W}$ de potencia. En un escenario de mínimo ahorro energético se evaluará el impacto de la sustitución de luminarias de vapor de sodio por luminarias LED sin ningún control de iluminación.

Por otro lado, en un escenario de máximo ahorro energético se evaluará el impacto de sustitución de luminarias de vapor de sodio por luminarias LED, con los niveles de iluminación correspondientes a las condiciones especificadas para los días laborables: lunes a viernes, y los fines de semana: sábados y domingos. La plataforma Cloud/GIS 
implementada permite regular los niveles de iluminación de las luminarias. Del $100 \%$ al $20 \%$, los días laborales de la semana, y del $50 \%$ al $10 \%$, los fines de semana. Las luminarias permanecen en funcionamiento 12 horas.

\subsubsection{Evaluación Energética y Económica para Luminarias Exteriores de la FISEI}

La Tabla 5, muestra una comparativa de cálculos obtenidos del consumo energético de 2 luminarias de vapor de sodio y 2 luminarias LED, en un escenario de mínimo ahorro energético, es decir, sin control de iluminación. Asimismo, se presentan importantes parámetros como el consumo energético, el coste económico de la iluminación del alumbrado público, el coste económico del sistema de iluminación con una proyección a 10 años y la cantidad de emisiones de $\mathrm{CO}_{2}$ por MWh, emitido por las luminarias. Según el Sistema Nacional Interconectado se estima que en Ecuador el factor de emisión de $\mathrm{CO}_{2}$ en el 2014 fue de 342,6 ( $\mathrm{g} \mathrm{CO}_{2} / \mathrm{kWh}$ ) (Narváez, R. P. 2015).

Las dos luminarias de vapor de sodio que se encuentran en la fachada frontal de la FISEI tienen un consumo energético anual de $2190 \mathrm{KWh} / \mathrm{año}$, con un coste económico de 137,97 dólares anuales. La vida útil de las luminarias de vapor de sodio es de hasta 16 mil horas y el costo de cada luminaria se estima en 290 dólares. Si se considera en un horizonte de 10 años, el cambio de luminarias se realizaría aproximadamente 3 veces. Así, el sistema de iluminación representaría un gasto económico igual a 3119,7 dólares.

Al implementar luminarias LED, el consumo energético anual equivale a $210,24 \mathrm{kWh}$, con un coste económico anual en iluminación pública de 13,24 dólares. Las luminarias LED de $24 \mathrm{~W}$ se estiman en un costo de 50 dólares, aproximadamente. El rendimiento de las luminarias LED se estima en 50 mil horas, así, en un horizonte de 10 años no se necesitaría cambio de luminarias y el coste del sistema de iluminación equivaldría a la inversión inicial y al coste económico de iluminación pública cada año, 232,45 dólares. Si se sustituyen luminarias de vapor de sodio por luminarias LED, se obtiene un ahorro tanto económico como energético de un $90 \%$.

Del mismo modo, en la Tabla 6, se muestran los cálculos obtenidos de consumo energético, coste económico de la iluminación pública, coste económico del sistema de iluminación con una proyección a 10 años y la cantidad de emisiones de $\mathrm{CO}_{2}$, para un escenario de máximo ahorro energético, con control de iluminación en luminarias LED. Las luminarias LED tienen una reducción del 100 al $20 \%$ en el nivel de iluminación, de lunes a viernes y del 50 al 10\%, los sábados y domingos.

La implementación de un sistema de control en las luminarias LED permite obtener un consumo energético anual de 126,14 $\mathrm{KWh}$, con un gasto total en el consumo eléctrico de iluminación anual equivalen a 7,94 dólares. El costo de los dispositivos de control se estima en unos 121 dólares por luminaria LED, con una vida útil de 3 años, así, en una proyección de 10 años, los dispositivos necesitarían ser cambiados 3 veces, estimando un costo total de 726 dólares, si le sumamos el costo de las luminarias LED y el costo económico de la iluminación pública, el costo del sistema de iluminación en 10 años equivaldría a un total de 905,47 dólares.

Con la implementación del sistema de control de iluminación sustituyendo luminarias de vapor de sodio en luminarias LED el ahorro económico anual obtenido es de aproximadamente un $94 \%$ y una reducción de 1433,34 toneladas de emisiones de $\mathrm{CO}_{2}$.

\subsubsection{Escenario Hipotético de Implementación de la Plataforma Cloud/GIS en el Campus Huachi UTA.}

La Universidad Técnica de Ambato, cuenta con aproximadamente 142 luminarias de vapor de sodio de 250 W. En la Tabla 7, se presentan los cálculos de consumo energético anual, coste económico de la iluminación pública, coste económico del consumo eléctrico del sistema de iluminación con una proyección a 10 años y la cantidad de emisiones de $\mathrm{CO}_{2}$, para un escenario de mínimo ahorro energético, con control de iluminación en luminarias LED. El sistema de control de iluminación permite reducir los niveles de porcentaje de iluminación del 100 al 20\%, de lunes a viernes y del 50 al 10\%, sábados y domingos. Las 142 luminarias de vapor de sodio de $250 \mathrm{~W}$ consumen un total de 155,49 MWh anuales, equivalente a un coste económico anual de iluminación pública de 9795,87 dólares.

En un horizonte de 10 años, el costo del sistema de iluminación equivaldría a un total de 221 mil dólares. Si se reemplazan las luminarias de vapor de sodio por luminarias LED, con una plataforma Cloud/GIS de control de nivel de iluminación, el consumo energético anual equivale a 8,9 MWh, con un coste anual en iluminación pública de 564,24 dólares. La inversión inicial del sistema de control de iluminación se estima en 24 mil dólares, pero el ahorro obtenido tanto económico como energético asciende a un $94 \%$. 
Tabla 5: Consumo energético y coste económico de luminarias del alumbrado público en un escenario de mínimo ahorro energético

\begin{tabular}{|c|c|c|c|c|c|c|}
\hline \multicolumn{7}{|c|}{ Mínimo ahorro energético/Sin Control de Iluminación } \\
\hline $\begin{array}{c}\text { Tipo de } \\
\text { Luminaria/Pot } \\
\text { encia } W\end{array}$ & $\begin{array}{l}\text { Consumo } \\
\text { energético } \\
\text { (kWh/día) }\end{array}$ & $\begin{array}{c}\text { Consumo } \\
\text { energético } \\
\text { anual } \\
(\mathbf{k W h} / \mathbf{a n ̃ o})\end{array}$ & $\begin{array}{l}\text { Coste económico } \\
\text { anual de } \\
\text { iluminación } \\
\text { publica } \\
\text { (USD) } \\
\end{array}$ & $\begin{array}{c}\text { Coste } \\
\text { económico } \\
\text { luminarias } \\
\text { (USD) }\end{array}$ & $\begin{array}{l}\text { Coste económico } \\
\text { Sistema de } \\
\text { iluminación en } 10 \\
\text { años } \\
\text { (USD) }\end{array}$ & $\begin{array}{c}\text { Emisión de } \mathrm{CO} 2 \\
\text { anual } \\
\left.\text { (Ton } \mathrm{CO}_{2} / \mathrm{kWh}\right)\end{array}$ \\
\hline $\begin{array}{c}\text { Vapor de Sodio } \\
2 \times 250 \mathrm{~W}\end{array}$ & 6 & 2190 & 137,97 & 580 & 3119,7 & 1520,96 \\
\hline $\begin{array}{c}\text { LED } \\
2 \times 24 \mathrm{~W}\end{array}$ & 0,576 & 210,2 & 13,25 & 100 & 232,45 & 146,01 \\
\hline
\end{tabular}

Tabla 6: Consumo energético y coste económico de luminarias del alumbrado público en un escenario de máximo ahorro energético

\begin{tabular}{|c|c|c|c|c|c|c|c|c|}
\hline \multicolumn{9}{|c|}{ Máximo ahorro energético } \\
\hline \multirow{2}{*}{$\begin{array}{c}\text { Tipo de } \\
\text { Luminaria/ } \\
\text { Potencia W }\end{array}$} & $\begin{array}{l}\text { Co } \\
\text { ene } \\
(\mathbf{k V}\end{array}$ & $\begin{array}{l}\text { mo } \\
\text { tico } \\
\text { lía) }\end{array}$ & \multirow{2}{*}{$\begin{array}{c}\text { Consumo } \\
\text { energético } \\
\text { anual } \\
\text { (kWh/año) }\end{array}$} & \multirow{2}{*}{$\begin{array}{l}\text { Coste } \\
\text { económico } \\
\text { anual de } \\
\text { iluminación } \\
\text { publica } \\
\text { (USD) }\end{array}$} & \multirow{2}{*}{$\begin{array}{c}\text { Coste } \\
\text { económico } \\
\text { luminarias } \\
\text { (USD) }\end{array}$} & \multirow{2}{*}{$\begin{array}{c}\text { Coste } \\
\text { económico } \\
\text { Sistema de } \\
\text { iluminación. } \\
\text { Luminarias+ } \\
\text { dispositivos de } \\
\text { control } \\
\text { (USD) } \\
\end{array}$} & \multirow{2}{*}{$\begin{array}{l}\text { Coste económico. } \\
\text { Sistema de } \\
\text { iluminación en } \\
10 \text { años } \\
\text { (USD) }\end{array}$} & \multirow{2}{*}{$\begin{array}{c}\text { Emisión de } \\
\text { CO2 anual } \\
\text { (Ton } \\
\left.\mathrm{CO}_{2} / \mathrm{kWh}\right)\end{array}$} \\
\hline & L-V & S-D & & & & & & \\
\hline $\begin{array}{c}\text { Vapor de Sodio } \\
2 \times 250 \mathrm{~W} \\
\text { LED }\end{array}$ & \multicolumn{2}{|c|}{6} & 2190 & 137,97 & 580 & $X$ & 3119,7 & 1520,96 \\
\hline $\begin{array}{l}\text { /Control } \\
2 \times 24 \mathrm{~W}\end{array}$ & 0,23 & 0,12 & 126,14 & 7,94 & 100 & 342 & 905,47 & 87,61 \\
\hline
\end{tabular}

Tabla 7: Consumo energético y coste económico del posible escenario de implementación del sistema de iluminación en el campus Huachi de la UTA.

\begin{tabular}{|c|c|c|c|c|c|c|c|c|}
\hline \multicolumn{9}{|c|}{ Escenario de iluminación en el campus Huachi de la UTA. } \\
\hline \multirow{2}{*}{$\begin{array}{c}\text { Tipo de } \\
\text { Luminaria/ } \\
\text { Potencia W }\end{array}$} & $\begin{array}{l}\text { Co } \\
\text { ene } \\
(\mathbf{k V}\end{array}$ & $\begin{array}{l}\text { mo } \\
\text { ico } \\
\text { lía) }\end{array}$ & \multirow{2}{*}{$\begin{array}{c}\text { Consumo } \\
\text { energético } \\
\text { anual } \\
(\mathbf{k W h} / \mathbf{a n ̃ o})\end{array}$} & \multirow{2}{*}{$\begin{array}{c}\text { Coste } \\
\text { económico } \\
\text { anual de } \\
\text { iluminación } \\
\text { publica } \\
\text { (USD) }\end{array}$} & \multirow{2}{*}{$\begin{array}{c}\text { Coste } \\
\text { económico } \\
\text { luminarias } \\
\text { (USD) }\end{array}$} & \multirow{2}{*}{$\begin{array}{c}\text { Coste } \\
\text { económico } \\
\text { Sistema de } \\
\text { iluminación. } \\
\text { Luminarias+ } \\
\text { dispositivos de } \\
\text { control } \\
\text { (USD) }\end{array}$} & \multirow{2}{*}{$\begin{array}{l}\text { Coste económico. } \\
\text { Sistema de } \\
\text { iluminación en } 10 \\
\text { años } \\
\text { (USD) }\end{array}$} & \multirow{2}{*}{$\begin{array}{c}\text { Emisión de } \\
\text { CO2 anual } \\
\text { (Ton } \\
\left.\mathrm{CO}_{2} / \mathrm{kWh}\right)\end{array}$} \\
\hline & L-V & S-D & & & & & & \\
\hline $\begin{array}{l}\text { Vapor de Sodio } \\
2 \times 250 \mathrm{~W}\end{array}$ & \multicolumn{2}{|c|}{426} & 155490 & 9795,87 & 41180 & $\mathrm{X}$ & 221498,7 & 107987,81 \\
\hline $\begin{array}{c}\text { LED } \\
/ \text { Control } \\
2 \times 24 \mathrm{~W}\end{array}$ & 16,35 & 8,17 & 8956,22 & 564,24 & 7100 & 24282 & 64288,4 & 6220,097 \\
\hline
\end{tabular}

\section{CONCLUSIONES}

La plataforma Cloud/GIS implementada permite regular los niveles de iluminación de las luminarias LED y obtener un ahorro adicional del consumo energético, además, de una reducción muy considerable en las pérdidas de energía eléctrica en los sistemas de distribución. Asimismo, la integración de un Sistema de Información Geográfica, GIS, en la Plataforma Cloud, permite la localización geográfica de los sistemas de control de iluminación, para un mayor control, de forma independiente, de cada uno o de clusters de sistemas de iluminación integrados.
La sustitución de las luminarias convencionales de vapor de sodio por luminarias con tecnología LED, es una alternativa eficiente para el sistema de alumbrado público. La implementación de luminarias LED permite un ahorro energético por KWh anual de hasta un $90 \%$.

La implementación de la plataforma Cloud/GIS en un sistema de iluminación pública con luminarias LED, en el Campus Huachi, UTA, se convierte en una solución tecnológica innovadora para el ahorro energético. La plataforma Cloud/GIS permitiría obtener un ahorro anual de hasta un $97 \%$, con un ahorro económico anual superior a 157 mil dólares en 10 años. 


\section{AGRADECIMIENTOS}

Los autores desean agradecer a la Dirección de Investigación y Desarrollo, DIDE de la Universidad Técnica de Ambato por su especial apoyo en el desarrollo de la presente propuesta gracias a la financiación del proyecto: "Diseño e Implementación de un Sistema Monitoreo Remoto para las Instalaciones Fotovoltaicas Aisladas de las Comunidades Amazónicas del Ecuador".

\section{REFERENCIAS}

Dangermond, J. (2012). Geography: A Platform for Understanding. Invited Paper, reprint from ArcNews, 7.

Gómez Delgado, M. (2016). La educación geográfica empleando las tecnologías de la información geográfica.

Guamán, J. I., Vargas, C. L., García, M. G., \& Ríos, A. (2017). Plataformas de Control Inteligente de Iluminación Interior integrados en Sistemas de Distribución LVDC. Revista Técnica Energía, 13(1), pp. 169-178. https://doi.org/10.37116/revistaenergia.v13.n1.2017 .19

Guamán, J., Guevara, D., Vargas, C., Ríos, A., \& Nogales, R. (2017). Solar manager: Acquisition, treatment and isolated photovoltaic system information visualization cloud platform. POWER, 42(170mA), 700Ma.

Jin, D., Hannon, C., Li, Z., Cortes, P., Ramaraju, S., Burgess, P., \& Shahidehpour, M. (2016). Smart street lighting system: A platform for innovative smart city applications and a new frontier for cybersecurity. The Electricity Journal, 29(10), 28-35.

Mahmoud, E., Hegazy, O., \& El-Dien, M. N. (2013). Integration of GIS and cloud computing for emergency system. International Journal of Engineering and Computer Science, 2(10), 28892893.

Manzano, S., Peña-Ortiz, R., Guevara, D., \& Ríos, A. (2014). Plataforma Cloud para monitoreo remoto de sistemas fotovoltaicos aislados en el Ecuador. Maskana, 5(Ed. Esp.).

Manzano, S., Peña, R., Guevara, D., \& Ríos, A. (2014, November). An Overview Of Remote Monitoring PV Systems: Acquisition, Storages, Processing And Publication Of Real-Time Data Based On Cloud Computing. In 13Th International Workshop on Large-Scale Integration of Wind Power into Power
Systems as well as on Transmission Networks for Offshore Wind Power Plants \& 4th Solar Integration Workshop which will be held from.

Manzano, S., Peña, R., Guevara, D., \& Ríos, A. (2014). A cloud scalable platform for monitoring isolated PV systems using wireless remote sensors in Ecuador. Proceedings ExtremeCom, 4-8.

Manzano, S., Guevara, D., \& Ríos, A. (2015). A new architecture proposal for PV remote monitoring based on IoT and cloud computing. Renewable Energy \& Power Quality Journal, RE\&PQJ.

Morales, M. F. (2012). Computación en la nube para automatizar unidades de información. Bibliotecas: Revista de la Escuela de Bibliotecología, Documentación e Información, 30(1).

Narváez, R. P. (2015). Factor de emisión de CO 2 debido a la generación de electricidad en el Ecuador durante el periodo 2001-2014. ACI Avances en Ciencias e Ingenierías, 7(2). C80-C85

Parkash, Prabu V, Dandu Rajendra. (2016). Internet of Things Based Intelligent Street Lighting System for Smart City. Departamento de Diseño de Sistemas Embebidos, NIELIT, Calicut India. 5(5).

Pisu, C., \& Casu, P. (2013). Cloud GIS and 3d modelling to enhance sardinian late gothic architectural heritage. International Archives of the Photogrammetry, Remote Sensing and Spatial Information Sciences, 5, W2. 489-494. 10.5194/isprsarchives-XL-5-W2-489-2013.

Ríos, A., Vargas, C., Guamán, J., \& Rodríguez, K. (2017). Sistema de Iluminación Fotovoltaico en el Alumbrado Público Gestionado a través de una Plataforma Cloud/GIS. Revista Politécnica, 39(1), 59-66.

Rodríguez, N. R., Murazzo, M. A., Chávez, S. B., \& Guevara, M. J. (2014). Arquitectura de cloud computing híbrida basada en tecnologías open source. In XX Congreso Argentino de Ciencias de la Computación (Buenos Aires, 2014).

Segura, A. A. (2016). Arquitectura de Software de Referencia para Objetos Inteligentes en Internet de las Cosas. Revista Latinoamericana de Ingenieria de Software, 4(2), 73-110.

Soni, E., Soni, V., \& Annapurna, D. (2016). Remotely Controlled Automated Street Lights: A Novel Approach towards IoT (Internet of Things), (April). 
Vargas, C., Guamán, J., Nogales, R., \& Ríos, A. (2017). Photovoltaic Lighting System with Intelligent Control based on ZigBee and Arduino. Energy, $100,190$.

Vargas, C., García, M., Guevara, D., \& Ríos, A. (2016). Escenarios de Integración de Sistemas Inteligentes de Iluminación Fotovoltaica en las Autopistas del Ecuador. Revista Técnica Energía, (12).

Vargas, C., Guevara, D., \& Ríos, A. (2014). Viabilidad económica del control de la iluminación fotovoltaica en autopistas del Ecuador.

Yoomak, S., Jettanasen, C., Ngaopitakkul, A., Bunjongjit, S., \& Leelajindakrairerk, M. (2018). Comparative study of lighting quality and power quality for LED and HPS luminaires in a roadway lighting system. Energy and Buildings, 159(December 2017), 542 557. https://doi.org/10.1016/j.enbuild.2017.11.060

\section{BIOGRAFÍAS}

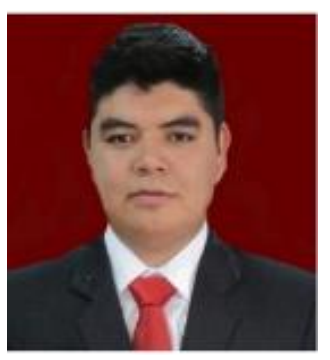

Carlos Luis Vargas GuevaraNació en Ambato, Ecuador en 1991. Ingeniero en Electrónica y Comunicaciones por la Universidad Técnica de Ambato, 2015. Asistente de Investigación en la Unidad Operativa de Investigación y Desarrollo de la Facultad de Ingeniería en Sistemas, Electrónica e Industrial de la Universidad Técnica de Ambato. En la actualidad se encuentra realizando sus estudios de Posgrado en la Escuela Politécnica Nacional en la Maestría en Electricidad mención Redes Eléctricas Inteligentes.

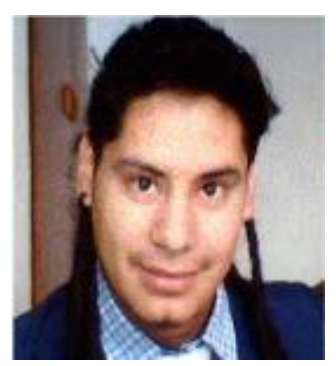

Jesús Guamán Molina- Nació en Latacunga, Ecuador en 1990. Ingeniero en Electrónica y Comunicaciones por la Universidad Técnica de Ambato, 2015. Desde el año 2015, trabaja en calidad de Asistente de Investigación en la Unidad Operativa de Investigación y Desarrollo de la Facultad de Ingeniería en Sistemas, Electrónica e Industrial de la Universidad Técnica de Ambato. En la actualidad se encuentra realizando sus estudios de Posgrado en la Escuela Politécnica Nacional en la Maestría en Electricidad mención Redes Eléctricas Inteligentes.

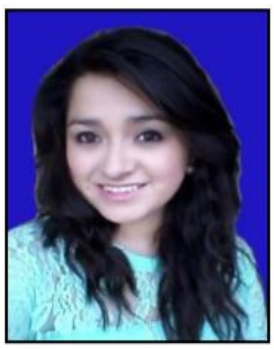

Katherine Rodríguez Mora. Nació en Ambato, Ecuador, el 26 de julio de 1992. Se graduó como bachiller en el Colegio "La Inmaculada" en el 2010. Recibió su título de Ingeniera en Electrónica y Comunicaciones en la Universidad Técnica de Ambato, UTA, 2016.

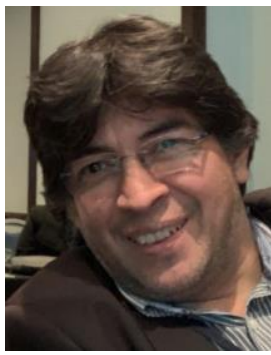

Alberto Ríos Villacorta- Dr. Ingeniero Eléctrico por Universidad Carlos III de Madrid, 2007. Master en Energías Renovables por la Universidad Europea de Madrid, 2004. Ingeniero Eléctrico en Sistemas y Redes Eléctricas por el Instituto Politécnico de Bielorrusia, 1993. Profesor Ayudante de la Universidad Carlos III de Madrid, 1998 - 2001. Profesor Adjunto de la Universidad Europea de Madrid, 2001-2014. Director Técnico del Laboratorio de Ensayos de Turbinas Eólicas y Simulaciones de parques Eólicos, Energy to Quality, 2005-2006. Director del Master Oficial de Energías Renovables de la Universidad Europea de Madrid, 2007-2011. Investigador Postdoctoral en la Universidad Técnica de Ambato entre 2014-2016, Beca Prometeo, SENESCYT, Ecuador. Profesor Titular Principal, Facultad de Ingeniería en Sistemas, Electrónica e Industrial, Universidad Técnica de Ambato, 2017. Consultor Internacional, Especialista en Energías Renovables y Eficiencia. 